\title{
A comparison of option prices under different pricing measures in a stochastic volatility model with correlation ${ }^{\dagger}$
}

\author{
Vicky Henderson $\ddagger$ \\ David Hobson $\S$ \\ Princeton University \\ University of Bath \\ Sam Howison ब \\ Tino Kluge \\ University of Oxford \\ University of Oxford
}

First version: March 20, 2003

First submitted: October, 2003

This version: October 6, 2004

\footnotetext{
${ }^{\dagger}$ We would like to thank participants at the Oxford-Princeton Mathematical Finance Workshop, especially Roger Lee and Peter Carr, the 6th Columbia-JAFEE Conference and seminar participants at Warwick Business School, Imperial College London, Heriot-Watt University, Humboldt University, University of Tokyo, Waseda University and Columbia University. The second author is supported by an Advanced Fellowship from the EPSRC. The fourth author acknowledges partial support from DAAD, EPSRC and KWI.

${ }^{\ddagger}$ Corresponding author. Department of Operations Research and Financial Engineering, and the Bendheim Center for Finance, Princeton University, Princeton, NJ, 08544. Email: vhenders@princeton.edu. Tel (609) 2587923

${ }^{\S}$ Department of Mathematics, University of Bath, Bath. BA2 7AY. UK

"Nomura Centre for Quantitative Finance, OCIAM, Mathematical Institute, 24-29 St. Giles', Oxford, OX1 3LB. UK.
} 
This paper investigates option prices in an incomplete stochastic volatility model with correlation. In a general setting, we prove an ordering result which says that prices for European options with convex payoffs are decreasing in the market price of volatility risk.

As an example, and as our main motivation, we investigate option pricing under the class of $q$-optimal pricing measures. The $q$-optimal pricing measure is related to the marginal utility indifference price of an agent with constant relative risk aversion. Using the ordering result, we prove comparison theorems between option prices under the minimal martingale, minimal entropy and variance-optimal pricing measures. If the Sharpe ratio is deterministic, the comparison collapses to the well known result that option prices computed under these three pricing measures are the same.

As a concrete example, we specialize to a variant of the Hull-White or Heston model for which the Sharpe ratio is increasing in volatility. For this example we are able to deduce option prices are decreasing in the parameter $q$. Numerical solution of the pricing pde corroborates the theory and shows the magnitude of the differences in option price due to varying $q$.

JEL Classification: D52, G13

Keywords: stochastic volatility, pricing measure, market price of volatility risk, Heston model, Hull White model 
Stochastic volatility models were developed as it became apparent that the Black Scholes option pricing formula exhibits pricing biases across moneyness and maturity. In particular, the Black Scholes formula underprices deep out-of-themoney puts and calls. Further, empirical evidence suggests that stock return distributions are negatively skewed with higher kurtosis than the lognormal distibution, see Bates (1996) and Bakshi et al (1997). The evidence for negative correlation between asset and volatility is particularly strong in the equity markets, see Nandi (1988) and Belledin and Schlag (1999). Stochastic volatility models provide a potential explanation of both the skew and kurtosis effects.

In an incomplete market model, such as a stochastic volatility model, there are no unique preference-independent prices for options. In recent years there has been much research in the area of characterizing pricing measures in incomplete markets. Of particular interest is the special case of option prices under stochastic volatility models, since such models exhibit incompleteness without the additional complication of jumps. This paper adds to this literature by presenting a comparison of option prices under various choices of pricing measures, or equivalently, various market prices of volatility risk.

The contribution of this article is threefold. First, in a general setting of an autonomous stochastic volatility model with correlation, we prove an ordering result that the prices of options with convex payoff structures are decreasing in the market price of volatility risk. This result should be compared with, and is an extension of, the results of Bergman et al (1996), El Karoui et al (1998), Hobson (1998) and Romano and Touzi (1997) which show that the option price is increasing in volatility. The fact that the prices of puts and calls are decreasing in the market price of volatility risk is intuitive, at least when the asset and its volatility are uncorrelated. However, this fact needs proof, especially in a model with non-zero correlation, where the result is less clear.

Second, we apply these results to the class of $q$-optimal pricing measures which have received much attention recently in the mathematical finance literature. The minimal entropy martingale measure (Fritteli, (2000)), the variance-optimal martingale measure (Föllmer and Sondermann, (1986)) and the minimal reverse entropy martingale measure (Schweizer, (1999)) are all special cases of $q$-optimal measures. The $q$-optimal measure is the risk-neutral or martingale measure which is closest to the physical or real world measure, where the notion of close depends on a dis- 
tance metric based on the $q^{\text {th }}$ moments of the relative density. Prices under the various $q$-optimal measures are the marginal (ie small quantity) utility-indifference bid prices for agents with HARA utilities, so our results can be interpreted in terms of utility maximizing agents.

Our goal is to prove comparison theorems between option prices under these various pricing measures and under the minimal martingale measure (Föllmer and Schweizer, (1991)). The analysis utilizes recent results of Hobson (2002) on characterizing $q$-optimal measures in stochastic volatility models.

For example we find that if the Sharpe ratio is deterministic then in our jump-free setting the $q$-optimal measures all collapse to the minimal martingale measure. This class of models is often described as being 'almost complete', see Schweizer (1999), and Pham et al (1998). More importantly, our paper analyzes option price orderings outside this special 'almost complete' case.

Our third contribution is to undertake a numerical investigation of our results in the Hull and White (1988) or Heston (1993) model. In this model we can write down explicit forms for many of the quantities of interest (including the market price of volatility risk, and the form of the $q$-optimal measure). One important conclusion is that the market price of volatility risk is not constant but depends on the level of volatility and maturity of the option to be priced. The numerical results support the theory by illustrating the fact that option prices are monotonic in the parameter $q$, and also provide evidence of the magnitude of the price changes with respect to $q$.

Our theoretical results can be seen as an extension of the results in Henderson (2002). Henderson studies the special case when there is no correlation between the asset and volatility. In her case, stronger ordering results are obtained, but only under the restrictive assumption of zero correlation. As we remarked above this is an unrealistic model in many markets. Our techniques also differ from Henderson (2002). We use partial differential equation (pde) arguments which generalize more simply to non-zero correlation than the coupling methods of Henderson.

Similarly our numerical results can be seen as extensions of the results of Heath et al (2001). These authors compare option prices under the variance-optimal $(q=2)$ and minimal martingale measure $(q=0)$ in the Heston model with zero correlation. Our paper extends their results to non-zero correlation and to arbitrary values of $q$. Indeed we show that the computations in Heath et al are incorrect. The 
numerics of Heath et al are in contradiction with our main theorem, whereas our numerics are consistent with our result. We cannot explain why their results are incorrect but it may follow from a false statement about put-call parity on p404.

The remainder of the paper is organized as follows. Section 1 begins by defining the class of stochastic volatility models under consideration in the paper and describes the form of the pricing measures. The general option price ordering result is stated and proved in Section 2. In the following section, we specialize to the class of $q$-optimal measures and summarize their properties. Section 4 employs the general ordering result together with the characterization of the $q$-optimal measures to compare option prices in a general stochastic volatility model. We can obtain stronger results by specializing to the Heston (1993) stochastic volatility model, and this is the subject of Section 5. Option prices are generated under the Heston model and their dependence on the choice of $q$ explained. Section 6 concludes the paper. Proofs and various technical remarks are relegated to a pair of appendices.

\section{Stochastic Volatility models}

Let $S$ be the price of the traded asset and let $V$ be the stochastic volatility. Under the assumption that the risk-free rate is non-stochastic, there is no loss of generality using discounted quantities, and $S$ represents the discounted asset price. In principle, $S$ and $V$ could be vector valued quantities, but in this paper we will only consider the univariate case. Under the real world measure $\mathbb{P}$ let $S$ and $V$ solve:

$$
\frac{d S_{t}}{S_{t}}=V_{t}\left(\alpha\left(t, V_{t}\right) d t+d B_{t}\right), \quad d V_{t}=a\left(t, V_{t}\right) d t+b\left(t, V_{t}\right) d W_{t},
$$

where $B, W$ are correlated Brownian motions with a constant correlation co-efficient $\rho$. We assume $\rho$ takes a value in $(-1,1)$ and we write $\bar{\rho}=\sqrt{1-\rho^{2}}$ so that $W$ can be represented via $d W_{t}=\rho d B_{t}+\bar{\rho} d Z_{t}$ where $Z$ is a Brownian motion which is independent of $B$.

We assume $V$ is a non-negative process, this covers the main models in the literature including Heston (1993) and Hull and White (1987, 1988). By convention, we take $b\left(t, V_{t}\right)>0$ throughout.

The model (1) is not the most general stochastic volatility model. For example it is possible to let $\alpha, a$ and $b$ be functions of $S$ as well as $V$ (or even to let them be non-Markovian) and to let $\rho$ depend on $t, S$ or $V$. However the framework (1) does include most of the standard stochastic volatility models in the literature and has 
the feature that the volatility process is an autonomous diffusion. It is this feature that will allow us to prove many of our results.

The price process $S$ in (1) has drift $\mu\left(t, V_{t}\right)=\alpha\left(t, V_{t}\right) V_{t}$ and volatility $V_{t}$. In such a parameterization, $\alpha\left(t, V_{t}\right)$ should be interpreted as the Sharpe ratio or equity risk premium ${ }^{1}$. The variable $\alpha\left(t, V_{t}\right)$ will play an important role in our comparisons.

Our analysis takes place within a model in which the probabilities of real world events are given by the physical measure $\mathbb{P}$. Note that in many option pricing papers the role of $\mathbb{P}$ is merely to determine the set of null events. Indeed sometimes the physical measure $\mathbb{P}$ is never specified and the author begins by writing down the dynamics under a pricing measure $\mathbb{Q}$. In our case, because we choose the pricing measure closest to $\mathbb{P}$, a different choice of real world measure $\mathbb{P}$ will lead to a different pricing measure $\mathbb{Q}$ and thus will have an effect on prices.

Since $S$ is the only traded asset in the model, and $V$ is not traded, it is not possible under the model in (1) to perfectly replicate a derivative on the stock price $S$. The market is incomplete, and potentially there are many probability measures under which the traded asset is a martingale. Denote the set of such measures by $\mathcal{Q}$. Each element of $\mathcal{Q}$ is a candidate pricing measure.

In the appendix we follow Frey (1997) to characterize the family of equivalent martingale measures. Each equivalent martingale measure is a candidate pricing measure. Roughly speaking a probability measure $\mathbb{Q} \in \mathcal{Q}$ is a martingale measure for $S$ on $\mathcal{F}_{T}$ if

$$
\left.\frac{d \mathbb{Q}}{d \mathbb{P}}\right|_{\mathcal{F}_{T}}=M_{T}
$$

where $M_{T}$ is the terminal value of a martingale $M_{t}$ given by

$$
M_{t}=\exp \left(\int_{0}^{t}\left[-\alpha\left(u, V_{u}\right) d B_{u}-\frac{1}{2} \alpha\left(u, V_{u}\right)^{2} d u-\lambda_{u} d Z_{u}-\frac{1}{2} \lambda_{u}^{2} d u\right]\right)
$$

for a suitable process $\lambda_{t}$.

The space of equivalent martingale measures is parameterized by the process $\lambda_{t}$ which governs the change of drift of $Z$. By Girsanov's theorem, under the change of measure $M_{T}$ we have two independent $\mathbb{Q}$-Brownian motions $B^{\mathbb{Q}}$ and $Z^{\mathbb{Q}}$ defined by

$$
d B_{t}^{\mathbb{Q}}=d B_{t}+\alpha\left(t, V_{t}\right) d t, \quad d Z_{t}^{\mathbb{Q}}=d Z_{t}+\lambda_{t} d t,
$$

\footnotetext{
${ }^{1}$ Put another way, we set $\alpha\left(t, V_{t}\right)=\frac{\mu\left(t, V_{t}\right)}{V_{t}}$ where $\mu\left(t, V_{t}\right)$ is the drift and $V_{t}$ the volatility. Since volatility is assumed positive, there is a one-to-one relationship between $\alpha$ and $\mu$.
} 
and then

$$
\begin{aligned}
\frac{d S_{t}}{S_{t}} & =V_{t} d B_{t}^{\mathbb{Q}} \\
d V_{t} & =\left[a\left(t, V_{t}\right)-\rho \alpha\left(t, V_{t}\right) b\left(t, V_{t}\right)-\bar{\rho} \lambda_{t} b\left(t, V_{t}\right)\right] d t+b\left(t, V_{t}\right) d W^{\mathbb{Q}}
\end{aligned}
$$

where $d W^{\mathbb{Q}}=\rho d B^{\mathbb{Q}}+\bar{\rho} d Z^{\mathbb{Q}}$.

Under $\mathbb{Q}$ the change of drift on $Z$ is $\lambda_{t}$, the associated change of drift on $W$ is $\rho \alpha\left(t, V_{t}\right)+\bar{\rho} \lambda_{t}$ and the change of drift on $V$ is $\left(\rho \alpha\left(t, V_{t}\right)+\bar{\rho} \lambda_{t}\right) b\left(t, V_{t}\right)$. The quantity

$$
\rho \alpha\left(t, V_{t}\right)+\bar{\rho} \lambda_{t}
$$

is termed the 'market price of volatility risk' or volatility risk premium. We sometimes call $\lambda_{t}$ the market price of $Z$ risk, also known in Lewis (2000) as the hedging portfolio risk premium. Note that since $\bar{\rho}$ is positive, then for a given model under $\mathbb{P}$, the market price of volatility risk is positively related to the market price of $Z$ risk.

We believe that given the representation of the risk neutral pricing measure $\mathbb{Q}$ via (2) and (3) it is most natural to model the parameter $\lambda_{t}$ and not to model the market price of volatility risk directly. This makes no difference when $\rho=0$, but when $\rho$ is non-zero arbitrarily choosing the market price of volatility risk to be constant (say) implies quite a complicated form for the risk neutral pricing measure.

One simple example of a candidate equivalent local martingale measure is the minimal martingale of Föllmer and Schweizer (1991). This is the measure which corresponds to the choice $\lambda \equiv 0$. Intuitively, under the minimal martingale measure the drifts of Brownian motions which correspond to traded assets are modified to make those assets into martingales, but the drifts of Brownian motions which are orthogonal to the traded assets are left unchanged.

We now turn to the question of option pricing within this model. A European option with non-negative payoff $h\left(S_{T}\right)$ can be priced by fixing $\mathbb{Q}$ in $\mathcal{Q}$ and then taking expectation under $\mathbb{Q}$. The time- $t$ option price $C\left(t, S_{t}, V_{t}\right)$ becomes

$$
C\left(t, S_{t}, V_{t}\right)=\mathbb{E}_{t}^{\mathbb{Q}} h\left(S_{T}\right)
$$

where the superscript $\mathbb{Q}$ refers to the fact that we are taking expectations with respect to $\mathbb{Q}$ and the subscript $t$ refers to the fact that we are conditioning on information at time $t$.

The advantages of fixing a martingale measure $\mathbb{Q}$ and using it for pricing are that the pricing functional is linear and that it agrees with the arbitrage free price for 
those options which can be replicated. Given the characterization above, selecting a particular $\mathbb{Q}$ is equivalent to choosing a market price of volatility risk, and the key to identifying the price is understanding the dynamics in (4).

\section{The Option Price Ordering Result}

This section proves our main ordering result which says that convex option prices are decreasing in the market price of $Z$ risk parameter $\lambda_{t}$, or equivalently decreasing in the market price of volatility risk. The intuition is that an increase in either $\lambda_{t}$ or the market price of volatility risk corresponds to a decrease in the drift of the volatility under the pricing measure.

Henceforth we only consider changes of drift $\lambda_{t}$ for the Brownian motion $Z$ which are Markov functions of the volatility process. Thus we suppose $\lambda_{t}=\lambda\left(t, V_{t}\right)$. We show in Section 3 that the market price of $Z$ risk for the $q$-optimal measure takes this form.

We begin this section by writing down a pair of stochastic volatility models under a pricing measure. (Note that we began the previous section by writing down a single model under the real world dynamics. The difference will be that under the pricing measure $S$ is a martingale.) Let the price process $S$ and volatility $V$ satisfy

$$
\frac{d S_{t}}{S_{t}}=V_{t} d B_{t} \quad d V_{t}=\eta\left(t, V_{t}\right) d t+b\left(t, V_{t}\right) d W
$$

where, as before, $d B d W=\rho d t$. Suppose that the drift on the volatility either takes the form ${ }^{2} \eta(t, v)=\eta^{+}(t, v)$ or $\eta(t, v)=\eta^{-}(t, v)$ and let $\mathbb{E}^{+}$(respectively $\mathbb{E}^{-}$) denote expectation with respect to the model with drift $\eta^{+}$(respectively $\eta^{-}$.) For a function $h$ define $J^{+}(t, s, v)=\mathbb{E}^{+}\left[h\left(S_{T}\right) \mid S_{t}=s, V_{t}=v\right]$, and similarly let $J^{-}(t, s, v)=\mathbb{E}^{-}\left[h\left(S_{T}\right) \mid S_{t}=s, V_{t}=v\right]$. Suppose that the diffusion co-efficients for both models satisfy the regularity conditions given in Appendix B.

Theorem 1 Consider the pair $(S, V)$ as defined in (5) and a convex function $h$. If $\eta^{+}(t, v) \geq \eta^{-}(t, v)$ for all $t$ and $v$, then

$$
J^{+}(t, s, v) \geq J^{-}(t, s, v)
$$

\footnotetext{
${ }^{2}$ The variables $\eta^{+}$and $\eta^{-}$are alternative specifications. The superscript + is used to denote the larger of the two functions and the minus is designed to represent the smaller quantity. These notations are not meant to represent the positive and negative parts of $\eta$.
} 
In particular, the prices of options with convex payoffs are higher under the model with drift $\eta^{+}$.

It is well known (see for example Bergman et al (1996)) that for calls and puts, option prices are increasing in the volatility process. This theorem is an extension of that result, but is similar in spirit. The volatility in the model with drift (on volatility) $\eta^{+}$is higher than the volatility in the model with drift $\eta^{-}$and, when combined with Jensen's inequality this leads to higher option prices. For convex payoffs, greater uncertainty (volatility) leads to higher option prices.

The proof of this result is given in Appendix A. There are two main ways in which Theorem 1 can be interpreted and applied. In both cases the theorem compares the prices of European-style options with convex payoffs. The first interpretation is to take the theorem literally and to compare two different models (with the same volatility structure but a different drift on the volatility process), where both models are specified under the pricing measure. The second interpretation, which we will use extensively later in the paper, is to consider a single model under a real-world measure $\mathbb{P}$ and to consider this model under two alternative specifications of equivalent martingale measure $\mathbb{Q}^{+}$and $\mathbb{Q}^{-}$.

In particular suppose the stochastic volatility model is as given in (1), and there are two candidate pricing measures defined via $\left(\lambda^{+}, \mathbb{Q}^{+}\right)$and $\left(\lambda^{-}, \mathbb{Q}^{-}\right)$respectively. Then if we set $\eta^{+}=a-\rho \alpha b-\bar{\rho} \lambda^{+} b$ and $\eta^{-}=a-\rho \alpha b-\bar{\rho} \lambda^{-} b$ and if $\lambda^{+}(t, v) \leq \lambda^{-}(t, v)$ then we have $\eta^{+} \geq \eta^{-}$. It follows from Theorem 1 that convex option prices are higher under the pricing measure $\mathbb{Q}^{+}$.

As an illustration, consider the example of the Hull and White (1987) model with non-zero correlation:

$$
\frac{d S_{t}}{S_{t}}=V_{t} d B_{t} \quad d V_{t}=a V d t+b V d W
$$

where $a$ and $b$ are constants. Then, if we compare two models with different values of parameter $a$, the expected payoffs of options (with convex payoffs) are higher in the model for which $a$ is higher. Further, if we consider the model (6) specified under $\mathbb{P}$, and if we consider the impact of pricing using a market price of volatility risk given by a constant $\lambda$, then the prices of options are decreasing in $\lambda$. 


\section{The Class of $q$-optimal pricing measures}

For the remainder of this paper, we focus on a subclass of the risk neutral pricing measures, namely the $q$-optimal measures. For a given $q$ the $q$-optimal measure is the equivalent martingale measure which is closest to the original real world measure $\mathbb{P}$ in the sense of the $q^{\text {th }}$ moment; see Delbaen et al (1997) and Hobson (2002). The $q$-optimal measure is also the pricing measure which gives the marginal prices for agents with HARA utilities who price by utility indifference.

In later sections we will compare option prices under stochastic volatility models of the form (1) where option prices are computed under various $q$-optimal measures. Note that in order to calculate the $q$-optimal measure it is necessary to know the real world dynamics and physical probability measure $\mathbb{P}$.

The fundamental idea is to choose a martingale measure $\mathbb{Q}$ as close as possible to $\mathbb{P}$. For $q \in \mathbb{R} \backslash\{0,1\}$ define

$$
H_{q}(\mathbb{P}, \mathbb{Q})=\left\{\begin{array}{cl}
\mathbb{E}\left[\frac{q}{q-1}\left(M_{T}\right)^{q}\right] & \text { if } \mathbb{Q} \ll \mathbb{P} \\
\infty & \text { otherwise }
\end{array}\right.
$$

and for $q \in\{0,1\}$ define

$$
H_{q}(\mathbb{P}, \mathbb{Q})=\left\{\begin{array}{cl}
\mathbb{E}\left[(-1)^{1+q} M_{T}^{q} \ln \left(M_{T}\right)\right] & \text { if } \mathbb{Q} \ll \mathbb{P} \\
\infty & \text { otherwise }
\end{array}\right.
$$

For $q \in \mathbb{R}$ the $q$-optimal measure is the measure $\mathbb{Q}^{(q)} \in \mathcal{Q}$ which minimizes $H_{q}(\mathbb{P}, \mathbb{Q})$.

A number of well studied martingale measures are special cases in the $q$-optimal class and, as such, this class provides a unifying framework. When $q=1, \mathbb{Q}^{(1)}$ is the minimal relative entropy measure, see Frittelli (2000). There are strong links between this pricing measure and pricing under exponential utility, see Delbaen et al (2002). Taking $q=0$ gives the minimal reverse entropy measure $\mathbb{Q}^{(0)}$. (The extra $\log$ terms when $q=0$ and $q=1$ can be related to $\log$ terms which arise when we integrate $x^{q-2}$ twice.) The case $q=2$ gives the variance-optimal measure, which is related to mean-variance hedging, see Föllmer and Sondermann (1986) and Duffie and Richardson (1991). In a stochastic volatility context, the variance optimal measure has been studied extensively by Laurent and Pham (1999), Biagini et al (2000) and Heath et al (2001).

The case $q=0$ has some special features. In a continuous setting such as ours (and under certain regularity assumptions) Schweizer (1999) has shown that 
the measure which minimizes the reverse relative entropy is precisely the (FöllmerSchweizer) minimal martingale measure. This corresponds to the choice $\lambda \equiv 0$ and implies that the non-hedgeable risk is not priced. We will recover this result in Corollary 4 below.

Although the cases $q=0,1,2$ have special properties, the criterion of choosing $\mathbb{Q}$ to minimize $H_{q}$ makes sense for all $q$ in $\mathbb{R}$. Further, for $q \neq 0,1$ there are links between pricing under the $q$-optimal measure and utility indifference pricing under a power-law utility. The cases $q<1$ correspond to strictly increasing, concave utility functions defined on $\mathbb{R}^{+}$. More specifically, for $q<1$, the price under the $q$-optimal measure corresponds exactly to the marginal utility indifference price for an agent with power utility $U(x)=\frac{x^{1-R}}{1-R}$ with constant relative risk aversion $R$ where $q=1-\frac{1}{R}$ or equivalently $R=\frac{1}{1-q}$. The case $q=0$ corresponds to $R=1$ and logarithmic utility.

To investigate the dependence of option prices on the choice of $q$-optimal measure, we need to be able to characterize such measures. Motivated by results in Rheinländer (2002) for the minimal entropy case, Hobson (2002) derives a representation equation which characterizes the $q$-optimal measure. It remains to solve the representation equation and to find the form of the market price of risk associated with the $q$-optimal measure. In the variance-optimal case with zero correlation, this was achieved by Laurent and Pham (1999) and Biagini et al (2000), and in the minimal entropy case within the Stein-Stein volatility model (with non-zero correlation) by Rheinländer (2002). Instead we follow Hobson (2002) who shows how to solve the $q$-optimal representation equation for models with correlation, at least for models of the form (1). In fact, he only considers $q \geq 1$ but his representation holds equally for $q<1$ so we are able to treat all the special cases of the general theory within the $q$-optimal setting. Defining $Q=1-q \rho^{2}$, the identification of the $q$-optimal measure $\mathbb{Q}^{(q)}$ is given via the market price of $Z$ risk,

$$
\lambda^{(q)}\left(t, V_{t}\right)=\bar{\rho} b\left(t, V_{t}\right) f_{v}\left(t, V_{t}\right)
$$

where $f=0$ if $q=0$ and otherwise

$$
f(t, v)=-\frac{1}{Q} \log \hat{\mathbb{E}}\left[\exp \left(-\frac{q}{2} Q \int_{t}^{T} \alpha\left(u, V_{u}\right)^{2} d u\right) \mid V_{t}=v\right]
$$

or

$$
f(t, v)=\hat{\mathbb{E}}\left[\frac{q}{2} \int_{t}^{T} \alpha\left(u, V_{u}\right)^{2} d u \mid V_{t}=v\right]
$$


in the special case of $Q=0$. Under $\hat{\mathbb{P}}, V$ has dynamics

$$
d V_{t}=\left(a\left(t, V_{t}\right)-q \rho \alpha\left(t, V_{t}\right) b\left(t, V_{t}\right)\right) d t+b\left(t, V_{t}\right) d \hat{W}
$$

with $\hat{\mathbb{P}}$-Brownian motion $\hat{W}$. If $\rho=0$ or $q=0$ then $\hat{\mathbb{P}}$ is just the real world measure $\mathbb{P}$.

By the Feynman-Kac theorem, $f$ solves the representation equation

$$
\frac{q}{2} \alpha(t, v)^{2}-q \rho b(t, v) \alpha(t, v) f_{v}-\frac{Q}{2} b(t, v)^{2}\left(f_{v}\right)^{2}+a(t, v) f_{v}+\frac{1}{2} b(t, v)^{2} f_{v v}+\dot{f}=0
$$

with $f(T, v)=0$.

Consider the representation (8). If $q Q>0$ (or equivalently, $q>0$ and $\rho^{2}<\frac{1}{q}$ ) then $f$ is positive and finite, and the $q$-optimal measure is defined over all time horizons. However, if $q Q \leq 0$, additional conditions are necessary to ensure the change of drift $\lambda^{(q)}$ is finite. Typically the function $f$ explodes at a finite timehorizon, beyond which the $q$-optimal measure ceases to be defined.

Under $\mathbb{Q}^{(q)}$, the dynamics in (4) become

$$
\begin{aligned}
\frac{d S_{t}}{S_{t}} & =V_{t} d B_{t}^{\mathbb{Q}^{(q)}} \\
d V_{t} & =\left[a\left(t, V_{t}\right)-\rho \alpha\left(t, V_{t}\right) b\left(t, V_{t}\right)-\bar{\rho}^{2} b\left(t, V_{t}\right)^{2} f_{v}\left(t, V_{t}\right)\right] d t+b\left(t, V_{t}\right) d W_{t}^{\mathbb{Q}^{(q)}}
\end{aligned}
$$

with $B_{t}^{\mathbb{Q}^{(q)}}=B_{t}+\int_{0}^{t} \alpha\left(u, V_{u}\right) d u, Z_{t}^{\mathbb{Q}^{(q)}}=Z_{t}+\int_{0}^{t} \bar{\rho} b\left(u, V_{u}\right) f_{v}\left(u, V_{u}\right) d u$ and $d W^{\mathbb{Q}^{(q)}}=$

$\rho d B^{\mathbb{Q}^{(q)}}+\bar{\rho} d Z^{\mathbb{Q}^{(q)}}$. These are the general model dynamics for the class of $q$-optimal measures.

\section{Comparisons between the $q$-optimal pricing mea- sures}

The general ordering theorem says option prices are decreasing in $\lambda$, or equivalently decreasing in the market price of volatility risk. Our task in this section is to analyze $\lambda^{(q)}$ for the $q$-optimal class of measures and hence to compare option prices. As we saw earlier, the $q$-optimal market price of $Z$ risk may be written as

$$
\lambda^{(q)}\left(t, V_{t}\right)=\bar{\rho} b\left(t, V_{t}\right) f_{v}\left(t, V_{t}\right)
$$

where $f$ is given in (8) or (9) and solves the pde (10). 
We first investigate the sign of $\lambda^{(q)}$. Under suitable regularity conditions (see Appendix B) we can prove the following theorem. The proof is again relegated to Appendix A.

Theorem 2 Under stochastic volatility dynamics in (1), and for $q \in \mathbb{R}$

(i) if $q \alpha(t, v)^{2}$ is non-decreasing in $v$, then $\lambda^{(q)} \geq 0$;

(ii) if $q \alpha(t, v)^{2}$ is non-increasing in $v$, then $\lambda^{(q)} \leq 0$;

(iii) the inequalities on $\lambda^{(q)}$ are strict if $q \alpha(t, v)^{2}$ is strictly increasing or decreasing in $v$.

Combining this result with the general ordering of Theorem 1 , and the fact that $\lambda^{(0)}=0$ we can make the following conclusion on the ordering of $q$-optimal option prices associated with convex payoffs.

Corollary 3 (i) Suppose for each $t, \alpha(t, v)^{2}$ is increasing in $v$. Then for $q>0$, option prices under the q-optimal measure are less than those under the minimal martingale measure, and for $q<0$, option prices under the q-optimal measure are greater than those under the minimal martingale measure. Equivalently, the marginal utility indifference prices for an agent with constant relative risk aversion $R>1$ are less than the marginal utility indifference prices for an agent with logarithmic utility, and for $R<1$ marginal prices are greater than those for logarithmic utility.

(ii) Conversely, suppose for each $t, \alpha(t, v)^{2}$ is decreasing in $v$. Then for $q>0$, option prices under the q-optimal measure are greater than those under the minimal martingale measure, and for $q<0$, option prices under the q-optimal measure are less than those under the minimal martingale measure. Equivalently, the marginal utility indifference prices for an agent with constant relative risk aversion $R>1$ are greater than the marginal utility indifference prices for an agent with logarithmic utility, and for $R<1$ marginal prices are less than those for logarithmic utility.

We can give a partial explanation for these results. Suppose $0<q<1$, or equivalently $1<R=\frac{1}{1-q}<\infty$ and that $\alpha$ is positive and increasing in $V$. If we take a mean-variance viewpoint, then as $R$ increases the agent is prepared to sacrifice some expected returns in order to keep variance (and especially losses) under control. Since the variance is driven by $\alpha^{2}$ which is increasing in $V$, the agent hedges against increases in $V$. Therefore under the state price density, the 
probability of scenarios with large volatility are reduced. If we now consider small ${ }^{3}$ options transactions, then the fact that volatilities are reduced means that the prices of options with convex payoffs are also reduced.

Corollary 4 If $\alpha$ is deterministic, then option prices under the q-optimal measure are all equal to the minimal martingale measure option price, and the marginal utility indifference price is the same for all agents with constant relative risk aversion, assuming they all share the same model of the dynamics under the physical measure.

In the light of Theorem 2, Corollary 3 and the results on the $\rho=0$ case of Henderson (2002), it is natural to make the following conjecture:

Conjecture 5 Option prices under the q-optimal measure are decreasing (respectively increasing) in $q$ if $\alpha^{2}$ is increasing (respectively decreasing) in $v$.

Although we cannot obtain a general result for the effect of $q$ on $\lambda^{(q)}$ when correlation is present, we can if we specialize to a particular model. In the next section we consider the Heston model.

\section{Option Price Comparisons under the square- root model}

In this section, we are interested in comparing various $q$-optimal option prices in the Hull and White (1988) or Heston (1993) model. In this special case we can give explicit formulæ for the market price of $Z$ risk (and hence market price of volatility risk) associated with the $q$-optimal measure. This allows us to analyze the effect of choice of $q$, or equivalently the choice of constant relative risk aversion co-efficient $R$ on option prices.

The Heston model gives the squared volatility $U$ as a squared Bessel process under the real world probability measure $\mathbb{P}$

$$
\begin{aligned}
\frac{d S_{t}}{S_{t}} & =\alpha\left(t, \sqrt{U_{t}}\right) \sqrt{U_{t}} d t+\sqrt{U_{t}} d B_{t}, \\
d U_{t} & =2 \kappa\left(m-U_{t}\right) d t+2 \beta \sqrt{U_{t}} d W_{t}
\end{aligned}
$$

\footnotetext{
${ }^{3}$ Here "small" means that to first order, entering this additional transaction does not affect the optimal investment portfolio
} 
with $B$ and $W$ correlated Brownian motions. Writing $U=V^{2}$ and $\bar{m}=m-\beta^{2} / 2 \kappa$ we get

$$
\frac{d S_{t}}{S_{t}}=V_{t}\left(\alpha\left(t, V_{t}\right) d t+d B_{t}\right) \quad d V_{t}=\kappa\left(\bar{m} / V_{t}-V_{t}\right) d t+\beta d W_{t} .
$$

This model is equivalent to taking $a\left(t, V_{t}\right)=\kappa\left(\bar{m} / V_{t}-V_{t}\right)$ and $b\left(t, V_{t}\right)=\beta$ in our original model. We assume $\beta, \kappa>0$ and $m \geq \beta^{2} / \kappa$, the latter to guarantee that $V$ does not hit zero.

The choice of the Sharpe ratio $\alpha\left(t, V_{t}\right)$ in the model will have a direct impact on the form of the $q$-optimal measure and hence the ordering of $q$-optimal option prices, via the function $f$ in (8) or (9). Before discussing the appropriate choice of $\alpha\left(t, V_{t}\right)$, we will describe the martingale measure used by Heston for pricing. In the original Heston (1993) model the Sharpe ratio term $\alpha\left(t, V_{t}\right)$ is of the form $\alpha\left(t, V_{t}\right)=\alpha_{H} / V_{t}$. To obtain a model under the risk neutral measure Heston adjusts the drift on the traded Brownian motion $B$ so that $S$ is a $\mathbb{Q}$-martingale and then proposes that the effect of the change of measure on the volatility $V$ is to adjust the drift by a term $\lambda^{\mathrm{H}} V$. Thus under the pricing measure the dynamics become

$$
\frac{d S_{t}}{S_{t}}=V_{t} d B_{t}^{\mathbb{Q}} \quad d V_{t}=\left\{\kappa\left(\bar{m} / V_{t}-V_{t}\right)-\lambda^{\mathrm{H}} V_{t}\right\} d t+\beta d W_{t}^{\mathbb{Q}} .
$$

In particular, although Heston makes the choice $\alpha\left(t, V_{t}\right)=\alpha_{H} / V_{t}$, his choice of pricing measure makes this term disappear. Note that the market price of volatility risk $\lambda^{\mathrm{H}} V / \beta$ is equivalent to a change of measure for which $d W^{\mathbb{Q}}=d W+\left(\lambda^{\mathrm{H}} V / \beta\right) d t$ is a $\mathbb{Q}$-martingale. In turn this corresponds to a change in drift on the orthogonal martingale $Z$ to make $Z^{\mathbb{Q}}$ given by $d Z^{\mathbb{Q}}=d Z-\frac{1}{\bar{\rho}}\left(\frac{\rho \alpha_{H}}{V}-\frac{\lambda^{\mathrm{H}} V}{\beta}\right) d t$ into a $\mathbb{Q}$-martingale. (The Brownian motions $B$ and $W$ are correlated, so although the switch to the pair $(B, Z)$ is merely a reparameterization, we contend that it is more natural to consider the effect of a change of measure in terms of the impact on the independent driving Brownian motions.)

Under the Heston change of drift,

$$
d V_{t}=\kappa^{*}\left(\frac{\bar{m}^{*}}{V_{t}}-V_{t}\right) d t+\beta d W_{t}^{\mathbb{Q}}
$$

where $\kappa^{*}=\kappa+\lambda^{\mathrm{H}}, \bar{m}^{*}=\frac{\kappa m-\frac{1}{2} \beta^{2}}{\kappa+\lambda^{\mathrm{H}}}$ are risk adjusted parameters and $W^{\mathbb{Q}}$ is a $\mathbb{Q}$-Brownian motion. Under this framework, Heston was able to obtain a pricing formula via Fourier inversion techniques involving numerical integration of the real 
part of a complex function. However, Heston's choice of pricing measure is essentially arbitrary and, in terms of its effect on the Brownian motion $Z$, not very natural. Certainly it is not one of the $q$-optimal measures. The choice does, however allow him to obtain a tractable method for pricing options.

Returning to the question of the specification of Sharpe ratio $\alpha\left(t, V_{t}\right)$ in the real world model (11), there are several possibilities. We disregard a constant Sharpe ratio $\left(\alpha\left(t, V_{t}\right)=\alpha_{0}\right)$ case since Corollary 4 implies option prices under all $q$ optimal measure prices are equal in this case. Heston (1993) takes $\alpha\left(t, V_{t}\right)=\alpha_{H} / V_{t}$, although as described, this has no impact in his model on option prices. The third choice of Sharpe ratio $\alpha\left(t, V_{t}\right)=\alpha_{1} V_{t}$ is a popular one in the literature, and appears as (H2) in Heath et al (2001), and as an example in both Hobson (2002) and Henderson (2002). If $\alpha_{1}>0$, this specification implies a Sharpe ratio which is increasing with volatility, a feature which is backed up empirically by Campbell and Cochrane (1997). In this section we will focus on the specification of the Heston model with $\alpha\left(t, V_{t}\right)=\alpha_{1} V_{t}$.

Hobson (2002, Proposition 5.1) gives an explicit formula for the solution of (8) and (9) (or equivalently (10)). In this case the solution to (10) is of the form

$$
f(t, v)=v^{2} F(T-t) / 2+G(T-t)
$$

where $F(0)=0=G(0)$ and $F$ and $G$ solve

$$
\dot{F}=q \alpha_{1}^{2}-2\left(\kappa+q \rho \beta \alpha_{1}\right) F-Q \beta^{2} F^{2}, \quad \dot{G}=\kappa m F .
$$

The differential equation for $F$ can be solved explicitly on a case by case basis depending on the signs of $q$ and $Q$. For example if $q$ and $Q$ are both positive,

$$
F(\tau)=\frac{C}{A} \tanh \left(A C \tau+\tanh ^{-1}\left(\frac{A B}{C}\right)\right)-B
$$

for suitable constants $A, B$ and $C$. This solution is finite for all time. For other parameter values the function $F$ may explode. It turns out that for any fixed correlation $\rho$ and maturity $T$ there exists $q_{*}<0<\rho^{-2}<q^{*}$ such that $F$ explodes before $T$ for $q$ outside the range $\left[q_{*}, q^{*}\right]$. If $F$ explodes then there is no $q$-optimal measure.

We are interested in the market price of $Z$ risk

$$
\lambda^{(q)}(t, V)=\bar{\rho} \beta V_{t} F(T-t)
$$


for the change of drift of $V$ under the $q$-optimal measure. Note the market price of risk is time-inhomogeneous.

We can now investigate the dependence of $\lambda^{(q)}$ on $q$ under the Heston model. The proof is in Appendix A.

Proposition 6 For the Heston model (11), with $\alpha\left(t, V_{t}\right)=\alpha_{1} V_{t}$, the market price of risk for the q-optimal pricing measure is increasing in $q$ for $q \in\left(q_{*}, q^{*}\right)$.

Combining Proposition 6 and the general comparison result in Theorem 1, we have (with $R_{*}=\frac{1}{1-q_{*}}<1$ )

Corollary 7 Consider the Heston model (11) with $\alpha\left(t, V_{t}\right)=\alpha_{1} V_{t}$. Over the range $\left(q_{*}, q^{*}\right)$ for which the q-optimal measure is well defined we have that q-optimal prices for European options with convex payoffs are decreasing in q. Further, over the range $\left(R_{*}, \infty\right)$, marginal utility indifference prices for European options with convex payoffs are decreasing in the co-efficient of relative risk aversion $R$.

This implies the highest option prices (within the class of $q$-optimal measure prices) are attained with the measures which correspond to the smallest values of $q$. In particular, the minimal martingale measure price is larger than the minimal entropy price, which in turn is greater than the variance-optimal price. Put another way, as the co-efficient of relative risk aversion increases, the marginal utility indifference price goes down.

Having established the general relationship between option prices and the choice of $q$ in the $q$-optimal measure in the Heston model, we now investigate numerically the magnitude of the differences in option prices. Our model parameters are appropriate for the foreign exchange market, although our qualitative conclusions do not depend on such a choice. Melino and Turnbull (1990) explore pricing in this market with stochastic volatility, as do Hakala and Wystup (2002). In the foreign exchange market, correlations tend to be small and positive. This differs from the equity market where strong negative correlations are the norm. The leverage effect is often cited to be the cause of this. As the stock price falls, debt to equity ratios rise making the firm riskier and resulting in greater volatility, see Nandi (1998).

In Figure 1, we show how the price of an at-the-money put option depends on the parameters $q$ and $\rho$. As anticipated by Corollary 7 , we observe the put price is decreasing in $q$. The range of the graph represents about a $16 \%$ difference in prices between the extreme points. If we examine special cases of moving from say $q=0$ 
Price

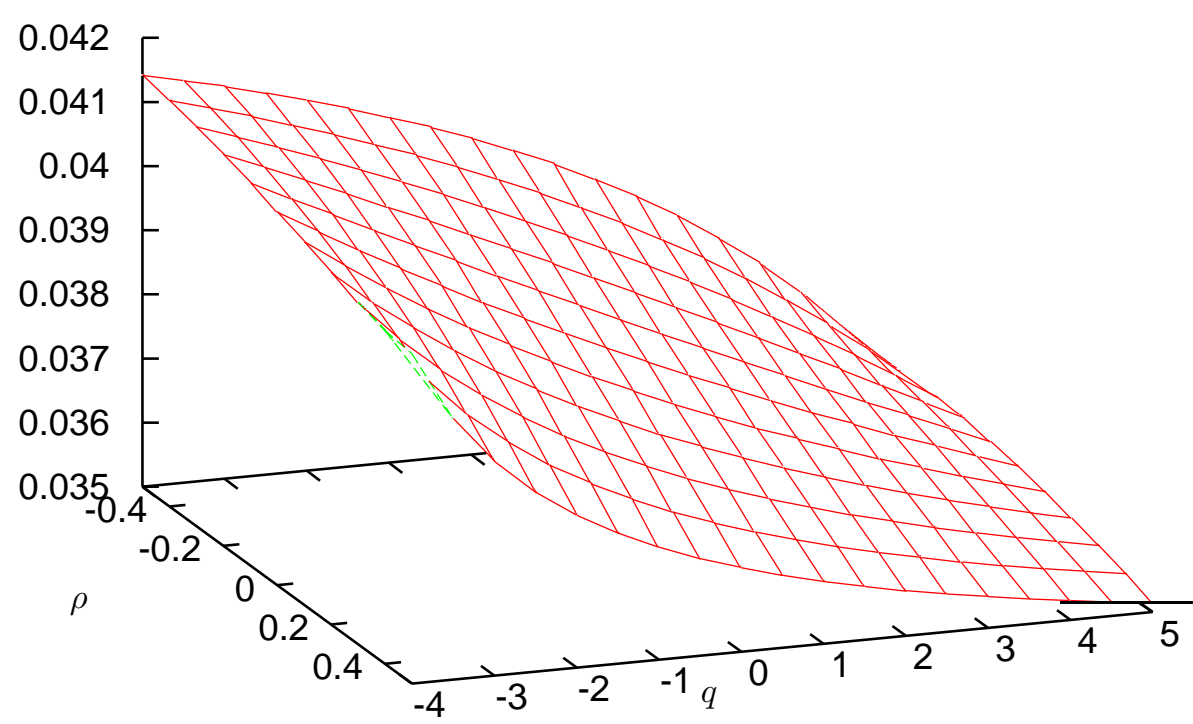

Figure 1: Price of a 1 year at-the-money put option as a function of the correlation $\rho$ between the asset price $S$ and the stochastic volatility $V$, and $q$, the parameter in the q-optimal measure related to the coefficient of relative risk aversion. The prices are computed under the Heston model where $d S_{t}=S_{t} V_{t}\left(\alpha_{1} V_{t} d t+d B_{t}\right)$ and $d V_{t}=\kappa\left(\bar{m} / V_{t}-V_{t}\right) d t+\beta d W_{t}$ with parameters as follows: $\kappa=1, \beta=0.1, \bar{m}=0.05, V_{0}=0.1, \alpha_{1}=4, S_{0}=1$. Since interest rates are zero, the prices of calls will equal the prices of puts. Over most of this range, $q\left(1-q \rho^{2}\right)>0$ and the q-optimal measure exists for all time, and even for $q=5$ and $|\rho|=0.5$ the $q$-optimal measure exists up to $T=1$. 
to $q=2$, the price change is of the order of a couple of percent, depending on the correlation. This is a non-trivial difference, and highlights the fact that choice of pricing measure or adjustment for risk has an impact on option prices.

In the figure, put option prices are also observed to decrease with correlation. It turns out that this is the case for 'small' $q$. Note that in the pricing pde (17), there are two drift terms arising from the incompleteness, $\rho \beta \alpha_{1} v$ and $\bar{\rho}^{2} \beta^{2} v F(T-t)$. In the small $q$ case, the first of these is dominant. If correlation is negative, this term has a positive effect on the option price, whilst the reverse is true for positive correlation. Once $q$ is no longer small, the ordering reverses. This is the case as the drift term involving $\bar{\rho}^{2} F(T-t)$ becomes dominant.

\section{Conclusion}

In this paper we have investigated the role of the market price of volatility risk and the choice of pricing measure on the prices of options. In a final section we compared these theoretical results for a general stochastic volatility model to numerical results for the Hull and White (1988) or Heston (1993) model.

Our first result is that as the market price of risk on the unhedgeable source of randomness $Z$ increases, or equivalently as the market price of volatility risk increases, the prices of European style options with convex payoffs decrease. At least when $\rho=0$, this result can be seen as an extension to an incomplete market of the standard result that in a complete market (such as the Black Scholes model) option prices are increasing in volatility.

As an application of this result we investigated how the market price of risk changes with $q$ when the pricing measure is chosen to be the $q$-optimal measure. If the innovations process driving the volatility is independent of that driving the traded asset then we recover the result of Henderson (2002) that if (the square of) the Sharpe ratio is increasing in volatility then (European) options with convex payoffs have prices decreasing in $q$. In the correlated case, the results are less clear, but we are able to show that under the same conditions, the $q$-optimal price for any positive $q$ is smaller than that for any negative $q$.

Since there is a direct relationship between the $q$-optimal measure and the marginal utility indifference price for an agent with HARA utility, these results have an immediate interpretation for an agent with constant relative risk aversion. 
For example, we can conclude that if $\rho=0$ and the Sharpe ratio is increasing in volatility, then the marginal utility indifference price of an agent with CRRA for a European option with convex payoff is decreasing in the risk aversion co-efficient $R$.

We conjecture that in the correlated case, if $\alpha(t, v)^{2}$ is increasing in $v$ then option prices are decreasing in $q$ or $R$ (so that the result for the correlated case is the same as when $\rho=0$ ). The evidence for the conjecture is based on the $\rho=0$ case and also on the analysis of the Heston model. However, this evidence is fairly slim, and it would be extremely interesting if it were possible either to prove the result or to find a counterexample. We hope this motivates the study of other examples, particularly if they lead to further explicit solutions, as this should help aid intuition.

Even when the correlation is zero we can have the following surprising result: namely that as an agent becomes more risk averse (which corresponds to $q$ increasing) $q$-optimal option prices may go down or up. The precise effect of changing $R$ depends on the Sharpe ratio.

An important observation is that the market price of volatility risk corresponding to the $q$-optimal measure is time-inhomogeneous. As a consequence, changing $q$ may have different effects for short and long maturity options. This additional feature of the model may provide extra ability for the model to fit market data and extra explanatory power. The issue of calibrating the model and inferring the parameter $q$ or risk aversion of the market is an extremely interesting one, and will be addressed elsewhere.

\section{Appendix A}

\section{Proof of Theorem 1}

The function $J^{+}$solves $\mathcal{L}^{+} J^{+}=0$ subject to $J^{+}(T, s, v)=h(s)$ where

$$
\mathcal{L}^{+}=\frac{1}{2} s^{2} v^{2} \frac{\partial^{2}}{\partial s^{2}}+\rho s v b(t, v) \frac{\partial^{2}}{\partial s \partial v}+\frac{b(t, v)^{2}}{2} \frac{\partial^{2}}{\partial v^{2}}+\eta^{+}(t, v) \frac{\partial}{\partial v}+\frac{\partial}{\partial t} .
$$

Similarly, under the alternative dynamics the function $J^{-}$solves $\mathcal{L}^{-} J^{-}=0$ subject to $J^{-}(T, s, v)=h(s)$ where $\mathcal{L}^{-}$is obtained from $\mathcal{L}^{+}$by replacing $\eta^{+}$with $\eta^{-}$.

As a consequence, for any function $g(t, s, v)$ we have $\mathcal{L}^{+} g-\mathcal{L}^{-} g=\left(\eta^{+}-\eta^{-}\right) g_{v}$. Here the subscript denotes a partial derivative. In general, a subscript $t$ or $u$ denotes a time parameter, other subscripts are partial derivatives, and the partial derivative with respect to time is denoted by a dot. 
Consider $\tilde{J}=J^{+}-J^{-}$so that $\tilde{J}(T, s, v)=0$. Then

$$
\mathcal{L}^{-} \tilde{J}=\mathcal{L}^{-}\left(J^{+}-J^{-}\right)=\mathcal{L}^{+} J^{+}-\mathcal{L}^{-} J^{-}-\left(\mathcal{L}^{+}-\mathcal{L}^{-}\right) J^{+}=-\left(\eta^{+}-\eta^{-}\right) J_{v}^{+} .
$$

By the Feynman-Kac representation

$$
\tilde{J}(t, s, v)=\mathbb{E}^{-}\left[\int_{t}^{T}\left(\eta^{+}-\eta^{-}\right) J_{v}^{+}\left(u, S_{u}, V_{u}\right) d u \mid S_{t}=s, V_{t}=v\right],
$$

and $\tilde{J} \geq 0$, provided $J_{v}^{+} \geq 0$.

It remains to show that the prices of options with convex payoffs are increasing in the initial value of the volatility, or equivalently that $J_{v}^{+} \geq 0$. This result can be found in the literature in a paper by Romano and Touzi (1997).

\section{Proof of Theorem 2}

If $q=0$ then $f \equiv 0$ and $\lambda^{(0)} \equiv 0$. Otherwise, consider first the case $Q \neq 0$. Under the transformation $g=e^{-Q f}, g$ is given by

$$
g(t, v)=\hat{\mathbb{E}} \exp \left(-\frac{q}{2} Q \int_{t}^{T} \alpha\left(u, V_{u}\right)^{2} d u\right)>0
$$

see (8), and (10) becomes

$$
-\frac{q}{2} Q \alpha(t, v)^{2} g+(a(t, v)-q \rho b(t, v) \alpha(t, v)) g_{v}+\frac{1}{2} g_{v v} b(t, v)^{2}+\dot{g}=0
$$

subject to $g(T, v)=1$. Now $g_{v}=-Q g f_{v}$ and we can examine the sign of $f_{v}$ via an analysis of $g_{v}$. The above pde can be written as

$$
0=\hat{\mathcal{L}} g-\frac{q}{2} Q \alpha(t, v)^{2} g
$$

where

$$
\hat{\mathcal{L}}=(a(t, v)-q \rho \alpha(t, v) b(t, v)) \frac{\partial}{\partial v}+\frac{1}{2} b(t, v)^{2} \frac{\partial^{2}}{\partial v^{2}}+\frac{\partial}{\partial t} .
$$

Differentiating (15) with respect to $v$ yields

$$
\begin{aligned}
0 & =\left[\hat{\mathcal{L}} g-\left(\frac{q}{2} Q \alpha(t, v)^{2}\right) g\right]_{v} \\
& =\hat{\mathcal{L}}_{v} g+\hat{\mathcal{L}} g_{v}-\frac{q}{2} Q\left(2 g \alpha \alpha_{v}+\alpha^{2} g_{v}\right) \\
& =\left(\left(a_{v}-q \rho b \alpha_{v}-q \rho \alpha b_{v}\right) \frac{\partial}{\partial v}+b b_{v} \frac{\partial^{2}}{\partial v^{2}}\right) g+\hat{\mathcal{L}} g_{v}-\frac{q}{2} Q\left(2 g \alpha \alpha_{v}+\alpha^{2} g_{v}\right) .
\end{aligned}
$$

Define

$$
\mathcal{L}^{\ddagger}=\hat{\mathcal{L}}+b(t, v) b_{v}(t, v) \frac{\partial}{\partial v} .
$$


Then $g_{v}(T, v)=0$ and

$$
0=\left(a_{v}-q \rho b \alpha_{v}-q \rho \alpha b_{v}-\frac{q}{2} Q \alpha^{2}\right) g_{v}+\mathcal{L}^{\ddagger} g_{v}-q Q \alpha \alpha_{v} g .
$$

By the Feynman-Kac representation,

$$
g_{v}(t, v)=-q Q \mathbb{E}^{\ddagger}\left[\int_{t}^{T} \alpha \alpha_{v} g \exp \left(\int_{t}^{s}\left(a_{v}-q \rho b \alpha_{v}-q \rho \alpha b_{v}-\frac{q}{2} Q \alpha^{2}\right) d u\right) d s\right]
$$

where under $\mathbb{P}^{\ddagger}, V$ has drift $\left(a-q \rho \alpha b+b b_{v}\right)$. Now recall $g_{v}=-Q g f_{v}$ so

$$
f_{v}=\frac{q}{g} \mathbb{E}^{\ddagger}\left[\int_{t}^{T} \alpha \alpha_{v} g \exp \left(\int_{t}^{s}\left(a_{v}-q \rho b \alpha_{v}-q \rho \alpha b_{v}-\frac{q}{2} Q \alpha^{2}\right) d u\right) d s\right] .
$$

Since $g>0$ we have that

$$
\begin{aligned}
& \text { if } q \alpha(t, v) \alpha_{v}(t, v)>0 \text { then } f_{v}>0 ; \\
& \text { if } q \alpha(t, v) \alpha_{v}(t, v)<0 \text { then } f_{v}<0 ; \\
& \text { if } q \alpha(t, v) \alpha_{v}(t, v)=0 \text { then } f_{v}=0 .
\end{aligned}
$$

It remains to consider the case $Q=0$. In that case (10) becomes

$$
\frac{q}{2} \alpha^{2}-q \rho f_{v} b \alpha+a f_{v}+\frac{1}{2} b^{2} f_{v v}+\dot{f}=0,
$$

subject to $f(T, v)=0$. Differentiating this equation with respect to $v$ and assuming that we can apply the Feynman-Kac formula we get

$$
f_{v}=q \mathbb{E}^{\ddagger}\left[\int_{t}^{T} \alpha(t, v) \alpha_{v}(t, v) \exp \left(\int_{t}^{s}\left(a_{v}-q \rho b \alpha_{v}-q \rho \alpha b_{v}\right) d u\right) d s\right]
$$

and the same conclusions.

\section{Proof of Proposition 7}

To decide the sign of $\left(\lambda^{(q)}\right)_{q}$ it is enough to know the sign of $f_{v q}$. Further, since $f(t, v)=v^{2} F(T-t) / 2+G(T-t)$ it is sufficient to know the sign of $H=F_{q}$ where $F(0)=0$ and $F$ solves (13). Differentiating (13) with respect to $q$ gives

$$
\dot{H}=\left(\alpha_{1}^{2}-2 \rho \beta \alpha_{1} F+\rho^{2} \beta^{2} F^{2}\right)-2\left(\kappa+q \rho \beta \alpha_{1}\right) H-2 Q \beta^{2} F H=\psi+\Theta H
$$

where $\psi=\left(\alpha_{1}^{2}-2 \rho \beta \alpha_{1} F+\rho^{2} \beta^{2} F^{2}\right) \geq 0$ and $\Theta=-2\left(Q \beta^{2} F+\kappa+q \rho \beta \alpha_{1}\right)$. Since $H(0)=0$ and $\dot{H}(0)=\alpha_{1}^{2}>0$ we conclude $H(t, V)>0$. 


\section{Appendix B}

In this appendix we make various technical remarks on the sufficient conditions which are necessary for our proofs to hold.

Our analysis takes place on a probability space $\left(\Omega, \mathcal{F},\left\{\mathcal{F}_{t}\right\}_{t \leq T_{+}}, \mathbb{P}\right)$ which supports the pair of independent Brownian motions $B$ and $Z$, and is such that these processes generate the filtration $\left(\mathcal{F}_{t}\right)$. $\mathbb{P}$ is the real world probability measure. The time $T_{+}$is a finite horizon time and we are interested in events up to the fixed time $T<T_{+}$where $T$ is the maturity of our option.

We need to assume that there exists a unique non-explosive strong solution to (1). Unfortunately, in the parameterization $(S, V)$ the standard conditions for existence and uniqueness of solutions to SDE's (e.g Rogers and Williams (1987, Theorem V 11.1) or Duffie (1996, Appendix E) do not apply. Instead to prove the necessary properties it is convenient to find a reparameterization $S=e^{X}$ and $V=\Upsilon(Y)$ for some pair $(X, Y)$ and a suitable function $\Upsilon$ for which the standard conditions apply. Once the existence and uniqueness of $(X, Y)$ has been proved, these properties will carry over to $S$ and $V$. We continue to work with $S$ and $V$ since these are the economically significant variables.

Recall that the market is incomplete, and there are many probability measures under which the traded asset is a (local) martingale. Each of these measures is a candidate pricing measure. Denote the set of such measures by $\mathcal{Q}_{l}$ and let the set of pricing measures under which $S$ is a true martingale be denoted by $\mathcal{Q}$. A necessary condition for the existence of any of these measures is that the Sharpe ratio satisfies $\int_{0}^{T} \alpha\left(u, V_{u}\right)^{2}<\infty$, almost surely, under the real world measure $\mathbb{P}$.

We follow Frey (1997) to characterize the family of equivalent martingale measures. A probability measure $\mathbb{Q} \in \mathcal{Q}_{l}$ equivalent to $\mathbb{P}$ on $\mathcal{F}_{T}$ is a local martingale measure for $S$ on $\mathcal{F}_{T}$ if and only if there is a progressively measurable process $\lambda=\left(\lambda_{t}\right)_{0 \leq t \leq T}$ and with $\int_{0}^{T} \lambda_{s}^{2} d s<\infty \mathbb{P}$ a.s., such that the local martingale $\left(M_{t}\right)_{0 \leq t \leq T}$ with

$$
M_{t}=\exp \left(\int_{0}^{t}\left[-\alpha\left(u, V_{u}\right) d B_{u}-\frac{1}{2} \alpha\left(u, V_{u}\right)^{2} d u-\lambda_{u} d Z_{u}-\frac{1}{2} \lambda_{u}^{2} d u\right]\right)
$$

satisfies $\mathbb{E} M_{T}=1$ and $M_{T}=\frac{d \mathbb{Q}}{d \mathbb{P}}$ on $\mathcal{F}_{T}$. The condition $\mathbb{E} M_{T}=1$ guarantees that $M$ is a true $\mathbb{P}$-martingale and that $\mathbb{Q}$ is a probability measure and $\mathbb{Q} \in \mathcal{Q}_{l}$. If $M_{t}$ is of form (3), then $S$ is in general only a $\mathbb{Q}$-local martingale. If $S$ is a true martingale 
then $\mathbb{Q} \in \mathcal{Q}$.

In Sections 2,3 and 4 we use the Feynman-Kac theorem (see Karatzas and Shreve (1991, Section 5.7B) or Duffie (1996, Appendix E)) to convert the solution of a Cauchy problem expressed via a pde into a stochastic representation. To use this result we need to know that the solution exists, and is unique (at least among the class of solutions satisfying a polynomial growth condition). The standard conditions for the existence and uniqueness of a solution (see Friedman (1975, p147), Karatzas and Shreve (1991) or Duffie (1996)) will not be satisfied in our parameterization. Again however, if $S=e^{X}$ and $V=\Upsilon(Y)$, and if the pair $(X, Y)$ satisfy appropriate regularity conditions, then the stochastic representation will hold. The appropriate conditions include the fact that the co-efficients of the SDE are differentiable and satisfy appropriate continuity, boundedness and growth conditions (above and below) and that the payoff function $h$ satisfies a growth condition. See for example the discussion in Romano and Touzi (1997) and especially the conditions (i) to (iv) on p401, or the paper by Heath and Schweizer (2000) which discusses more generally the connection between pdes and the martingale approach in finance. Note that these conditions are not satisfied by the Heston model but in that case it is possible to justify the stochastic representations directly without recourse to the pde approach: see (7) and (8). We also assume that the co-efficients of the diffusion processes are sufficiently smooth so that we can differentiate the related infinitesimal generators.

We now turn to the question of existence of $q$-optimal measures. This question has been dealt with by a number of authors. When $q=1$, Rheinländer (2002) gives a necessary and sufficient condition for the existence of an equivalent measure with finite relative entropy. A result of Frittelli (2000) then guarantees the minimal entropy measure exists. When $q=2$, existence is dealt with in Delbaen and Schachermayer (1996). In the particular setting of our stochastic volatility model, and given certain smoothness and boundedness conditions on the parameters, Hobson (2002), shows that if $q\left(1-q \rho^{2}\right)>0$ then the $q$-optimal measure always exists. If, on the other hand $q\left(1-q \rho^{2}\right) \leq 0$ then it may be the case that the $q$-optimal measure ceases to exist beyond a certain $q$-dependent time horizon. Hobson (2002) also has a discussion of a set of sufficient conditions which ensure that the analysis of Section 3 is valid.

Finally we make some remarks on the computations in Section 5. The numerical 
solutions are generated by solving the Heston pricing pde

$$
\begin{aligned}
& \dot{C}+C_{v}\left(\kappa(\bar{m} / v-v)-\rho \beta \alpha_{1} v-\bar{\rho}^{2} \beta^{2} v F(T-t)\right) \\
& +\frac{1}{2}\left(C_{s s} s^{2} v^{2}+C_{v v} \beta^{2}+2 C_{s v} s v \beta \rho\right)=0 \quad C(T, s, v)=h(s)
\end{aligned}
$$

using a Crank-Nicolson type finite difference method. We follow Kluge (2002) and apply this scheme to the $\log$ spot transformed pde $(x:=\log s)$ which is of convection diffusion type. At the zero volatility boundary, $v=0$, the diffusion term disappears and only the convection remains. With the restriction $m \geq \beta^{2} / \kappa$, which implies that the stochastic volatility process does not hit zero, it turns out that this is an outflow boundary. That is why no boundary conditions can be imposed and we use an upwind scheme at this boundary. All other boundaries are artificial due to the fact that the log spot transformed pde lives in $(x, v) \in \mathbb{R} \times \mathbb{R}_{+}$and have to be set sufficiently far away from the area of interest. To further reduce the numerical error a non-uniform grid in space and time dimension is used. Numerical accuracy of this scheme was examined in Kluge (2002) for $q=0$ and $\alpha_{1}=0$ and verified against the Heston closed form solution. 


\section{References}

Bakshi G., Cao C. and Z. Chen, 1997, "Empirical Performance of Alternative Option Pricing Models", Journal of Finance, 52, 2003-2049.

Bates D., 1996, "Testing Option Pricing Models", In G.S. Maddala and C.R. Rao (eds): Handbook of Statistics, Vol 15: Statistical Methods in Finance, North Holland Amsterdam, 567-611.

Belledin M. and C. Schlag, 1999, "An Empirical Comparison of Alternative Stochastic Volatility models", Preprint, Johann Wolfgang Goethe-Universität.

Bergman Y.Z., B.D. Grundy and Z. Wiener, 1996, "General Properties of Option Prices", Journal of Finance, 51, 5, 1573-1610.

Biagini F., P. Guasoni and M. Prattelli, 2000, "Mean Variance Hedging for Stochastic Volatility models", Mathematical Finance, 10(2), 109-123.

Campbell J. and Cochrane J.H., 1997, "By Force of Habit: A Consumption-based explanation of Aggregate Stock Market Behavior". Working paper.

Delbaen F., Grandits P., Rheinländer T., Samperi D., Schweizer M. and Stricker C., 2002, "Exponential hedging and entropic penalties", Mathematical Finance, 12(2), $99-123$.

Delbaen F., Monat P., Schachermayer W., Schweizer M., Stricker C., 1997, "Weighted Norm Inequalities and hedging in Incomplete Markets", Finance and Stochastics, $1,181-227$.

Delbaen F. and W. Schachermayer, 1996, "The Variance-Optimal martingale measure for continuous processes", Bernoulli, 2, 81-105, amendments and corrections Bernoulli, 2, 379-380.

Duffie D., 1996, "Dynamic Asset Pricing Theory". Princeton University Press, Second Edition.

Duffie D. and Richardson H.R., 1991, "Mean-Variance Hedging in Continuous Time", Annals of Applied Probability, 1, 1-15.

El Karoui N., M. Jeanblanc and S.E. Shreve, 1998, "Robustness of the Black Scholes Formula", Mathematical Finance, 8(2), 93-126. 
Föllmer H. and M. Schweizer, 1991, "Hedging of Contingent claims under incomplete information", In Applied Stochastic Analysis, Stochastic Monographs, Vol 5, eds M.H.A Davis and R.J.Elliot, New York, Gordon and Breach, 389-414.

Föllmer H. and Sondermann D., 1986, "Hedging of non-redundant contingent claims". In Contributions to Mathematical Economics, eds W. Hildenbrand and A. MasColell, Amsterdam: North Holland, 205-223.

Frey R., 1997, "Derivative Asset Analysis in models with level-dependent and stochastic volatility". CWI Quarterly, 10(1), 1-34.

Friedman A., 1975, "Stochastic Differential Equations and Applications, Vol.1", New York: Academic Press.

Frittelli M., 2000, "The minimal entropy martingale measure and the valuation problem in incomplete markets", Mathematical Finance, 10, 39-52.

Hakala J. and U. Wystup, 2002, "Heston's Stochastic Volatility Model applied to Foreign Exchange Options". In Foreign Exchange Risk: Models, Instruments and Strategies, eds: J. Hakala and U. Wystup, RISK Books, Chapter 23.

Heath D., E. Platen and M. Schweizer, 2001, "A Comparison of two Quadratic Approaches to Hedging in Incomplete Markets", Mathematical Finance, 11(4), 385413.

Heath D. and M. Schweizer, 2000, "Martingales versus pdes in finance: an equivalence result with examples", J. Applied Probability, 37, 947-957.

Henderson V., 2002, "Analytical Comparisons of Option Prices in Stochastic Volatility Models", Mathematical Finance, To appear.

Heston S.L., 1993, "A Closed form solution for options with stochastic volatility with applications to bond and currency options", Review of Financial Studies, 6, 327-343.

Hobson D., 1998, "Volatility Mis-specification, Option Pricing and Super-replication via Coupling", Annals of Applied Probability, 8(1), 193-205.

Hobson D., 2002, "Stochastic Volatility models, correlation and the $q$-optimal measure", Mathematical Finance, To appear.

Hull J.C and A. White, 1987, "The Pricing of Options on Assets with Stochastic Volatilities", Journal of Finance, 42, 281-300. 
Hull J.C. and A. White, 1988, "An Analysis of the Bias in Option Pricing caused by a Stochastic Volatility", Advances in Futures and Options Research, 3, 29-61.

Karatzas I. and S. Shreve, 1991, "Brownian motion and stochastic calculus", SpringerVerlag New York, Second Edition.

Kluge T., 2002, "Pricing Derivatives in Stochastic Volatility Models using the Finite Difference Method", Diploma Thesis, Technische Universität Chemnitz.

Laurent J.P and H. Pham, 1999, "Dynamic programming and mean-variance hedging", Finance and Stochastics, 3, 83-101.

Lewis A.L., 2000, "Option Valuation Under Stochastic Volatility: with Mathematica Code", Finance Press.

Melino A. and S. Turnbull, 1990, "The Pricing of Foreign Currency Options with Stochastic Volatility", Journal of Econometrics, 45, 239-265.

Nandi S., 1988, "How Important is the Correlation between Returns and Volatility in a Stochastic Volatility Model? Empirical Evidence from Pricing and Hedging in the S\&P500 Index Options Market", Journal of Banking and Finance, 22, 589-610.

Pham H., T. Rheinländer and M. Schweizer, 1998, "Mean-variance hedging for continuous processes: new proofs and examples", Finance and Stochastics, 2(2), 173-198.

Rheinländer T., 2002, "An entropy approach to stochastic volatility models", Preprint, ETH Zurich.

Rogers L.C.G. and Williams D., 1987, "Diffusions, Markov Processes and Martingales: Itô Calculus", Wiley.

Romano M. and Touzi N., 1997, "Contingent Claims and Market Completeness in a Stochastic Volatility Model", Mathematical Finance, 7 (4), 399-410.

Schweizer M.,1999, "A minimality property of the minimal martingale measure", Statistics and Probability Letters, 42, 27-31. 